\title{
Endometriosis en la pared abdominal
}

\section{Endometriosis of the abdominal wall}

\author{
Julio Enríquez-Merino, ${ }^{*}$ José Alberto Ramos-Garibay, ${ }^{\ddagger}$ \\ Mario Rodríguez Y-Silva, ${ }^{\S}$ Nayeli Meza-Gaxiola $₫$
}

\section{RESUMEN}

La endometriosis es una enfermedad ginecológica relativamente frecuente, inflamatoria, crónica, que se caracteriza clínicamente por la presencia de implantes de tejido endometrial fuera del útero. En muy raras ocasiones la endometriosis se ubica fuera de la cavidad pélvica formando endometriomas que se implantan en cualquier sitio. Este padecimiento ocasiona dolor cíclico pélvico, crónico e infertilidad en un alto porcentaje de casos en las mujeres que lo padecen. Hasta la fecha se desconoce su etiología, aunque se postulan varias teorías. No todas pueden explicar los diferentes tipos de endometriosis. El tratamiento es complejo y se basa en métodos no invasivos e invasivos. El objetivo de comunicar este caso es tener presente el diagnóstico de endometriosis al detectar cualquier masa palpable dolorosa en la pared abdominal en mujeres en edad fértil, ya que esta localización se observa muy raramente en el campo de la cirugía dermatológica.

Palabras clave: Endometriosis, endometriomas, masa palpable en la pared abdominal.

\section{INTRODUCCIÓN}

Se define a la endometriosis como la presencia de tejido endometrial (producto tanto del estroma como de las glándulas endometriales secretoras de tipo apocrino, cilíndricas, las cuales producen secreción por «decapitación») fuera de la cavidad uterina. Se le considera una enfermedad ginecológica relativamente frecuente, inflamatoria crónica, estrógeno dependiente.

\footnotetext{
* Jefe del Servicio de Cirugía Dermatológica.

₹ Dermatopatólogo.

§ Dermatólogo.

" Médico residente del segundo año de Dermatología.
}

Centro Dermatológico «Dr. Ladislao de la Pascua», SSCDMX.

\section{ABSTRACT}

Endometriosis is a frequent gynecological, chronic and inflammatory disease characterized by implants of endometrial tissue outside the uterus. Rarely, endometriosis can be located in the abdominal cavity as endometriomas. Endometriosis causes chronic pelvic cyclic pain, and infertility in a high percentage of cases of women who suffer from it. To date its etiology is unknown and several theories have been proposed, because none of them can explain the different types of endometriosis. Treatment is complex and is based on non-invasive and invasive methods. The objective of reporting this case is to bear in mind the diagnosis of endometriosis when detecting any palpable painful mass in the abdominal wall in women of childbearing age, since this location is very rarely observed in the field of dermatological surgery.

Keywords: Endometriosis, endometriomas, endometriosis of the abdominal wall.
Citar como: Enríquez-Merino J, Ramos-Garibay JA, Rodríguez Y-Silva M, MezaGaxiola N. Endometriosis en la pared abdominal. Rev Cent Dermatol Pascua. 2021; 30 (1): 24-28. https://dx.doi.org/10.35366/100588
Fue descrita por primera vez en 1860 por el médico y patólogo austriaco Von Rokitansky. ${ }^{1-3}$

Hasta la fecha se desconoce la causa exacta de la endometriosis. Existen varias teorías acerca de su etiopatogenia, sin embargo, ninguna de éstas explica todos los tipos descritos. La teoría más aceptada que intenta explicar el desarrollo de los implantes peritoneales es la de la menstruación retrógrada (teoría de Sampson). ${ }^{3}$ En ella se sugiere que las células del endometrio se transportan durante la menstruación, por reflujo, en dirección retrógrada hacia las trompas de Falopio, hasta llegar a la pelvis, en donde se instalan en las superficies serosas de ésta. ${ }^{3}$ 
La evidencia que apoya esta teoría se basa en que las mujeres afectadas generalmente producen una mayor cantidad de descarga hemática durante las menstruaciones, la cual sufre reflujo, en comparación con las que no desarrollan endometriosis. ${ }^{3}$ No obstante, se sabe que hasta $90 \%$ de las mujeres pueden tener menstruación retrógrada y sólo $15 \%$ de éstas desarrolla endometriosis confirmada. ${ }^{3}$

La teoría de la metaplasia celómica, o la teoría de Robert Meyer, consiste en la transformación metaplásica de las células que recubren el peritoneo visceral abdominal en tejido endometrial, debido a un estímulo hormono-ambiental. ${ }^{3}$ Otra de las teorías que aún se analiza es la embrionaria Mülleriana, en la que se propone que la endometriosis se basa en una alteración de las estructuras del tracto genital durante la organogénesis, lo cual permite que se desarrolle tejido endometrial fuera de la cavidad uterina, expresando estrógenos y CA $125 .^{3}$

La teoría de la diseminación vascular o linfática explica los implantes de tejido endometrial en sitios extrapélvicos, como nódulos linfáticos abdominales, pulmón, pleura y cerebro; sin embargo, no explica las lesiones pélvicas comunes. ${ }^{2-4} \mathrm{Si}$ se habla de trasplante directo, el antecedente de alguna intervención quirúrgica pélvica previa explica su presencia cuando se descubre este tipo de tejido en cicatrices abdominales. ${ }^{3}$ Otra hipótesis propuesta en la literatura es la teoría de las células madre, en la cual se sugiere que células indiferenciadas tienen la capacidad de generar depósitos endometriales. La teoría de la disfunción inmune menciona que las mujeres que cursan con alguna enfermedad autoinmune durante la menstruación desarrollan mayor inflamación, con la subsecuente eliminación deficiente de desechos endometriales, induciendo la formación de un endometrio ectópico. ${ }^{3}$

La endometriosis tiene una alta prevalencia que va de 10 a $20 \%$ en mujeres en edad fértil ( 25 a 44 años) y se incrementa hasta $40-80 \%$ en mujeres con el antecedente de dolor pélvico crónico o infertilidad, siendo éstos los principales motivos de consulta..$^{1-3}$ Los sitios más frecuentes de afectación son los ovarios, el peritoneo pélvico, los ligamentos uterosacros, las trompas de Falopio y el ligamento ancho. Menos frecuente es el compromiso del colon, el intestino delgado, el apéndice, la cicatriz umbilical $u$ otros tejidos contiguos a la cavidad pélvica. ${ }^{3,4}$ Se han publicado algunos casos en los que se ha detectado este tipo de tejido en el sistema nervioso central (SNC) o la mucosa nasal. ${ }^{5-7}$

A pesar de que la endometriosis es frecuente, y su diagnóstico no es inusual en la práctica ginecológica, la forma extrapélvica es rara y puede ser un hallazgo durante cualquier procedimiento quirúrgico, incluso en la práctica de la cirugía dermatológica. Desde hace más de 40 años se han comunicado casos de esta variedad, con una prevalencia de hasta $12 \% .{ }^{5}$ Se sugiere que es subdiagnosticada.

La endometriosis cutánea es la siembra de tejido endometrial en la pared abdominal. Cuando se manifiesta como una masa bien circunscrita se le denomina endometrioma. A nivel cutáneo, sin el antecedente de cirugía uterina previa, el desarrollo de éstos es poco frecuente. La incidencia varía de 1 a $2 \%$. La mayoría de los reportes de endometriosis de la pared abdominal (la cual es el sitio más frecuente de enfermedad extrapélvica) se asocian con el antecedente de cicatrices por cesárea u otros procedimientos quirúrgicos. ${ }^{4,6}$

La presentación clínica varía dependiendo del sitio afectado. ${ }^{4}$ Los síntomas más frecuentes en este tipo de endometriosis son la presencia de una masa abdominal subcutánea palpable (99\%), el dolor cíclico, que frecuentemente es atípico (71\%) y el sangrado cíclico $(48 \%) .{ }^{4,6} \mathrm{El}$ dolor en la endometriosis de la pared abdominal puede no ser un síntoma clínico constante y no asociado al ciclo menstrual, por lo que el diagnóstico puede ser difícil.

\section{DIAGNÓSTICO}

No existen estudios de laboratorio específicos para realizar el diagnóstico de endometriosis. La concentración sérica de CA 125 (proteína que se encuentra en la superficie de algunas células y que se considera un marcador tumoral) puede estar elevada (es decir, más de 35 unidades $/ \mathrm{mL}$ ) en algunas afecciones benignas, como es el caso de las mujeres con endometriosis. Su determinación inicial dentro del plan de abordaje diagnóstico no está aún bien definida. No se solicita de manera rutinaria en mujeres que están siendo evaluadas o tratadas por endometriosis, ya que otros padecimientos, en particular el carcinoma de ovario, endometrial, peritoneal o de las trompas de Falopio, también elevan la concentración sérica de esta proteína. ${ }^{3}$

Desde luego los hallazgos en una tomografía axial computarizada o una resonancia magnética orientan en gran medida al diagnóstico al evidenciar la presencia de masas en las diferentes estructuras orgánicas. Esta última diagnosticará con precisión la endometriosis torácica hasta en $95 \%$ de los casos.

La ultrasonografía en la endometriosis de la pared abdominal muestra una masa hipoecoica, vascular y/o sólida. Los márgenes son irregulares, a menudo espiculados, y puede parecer que se infiltran en los tejidos adyacentes. 
Una ecografía transvaginal muestra imágenes que sugieren endometriosis pélvica, con endometriomas en los ovarios, nódulos en el tabique rectovaginal e incluso en la vejiga. ${ }^{7-9}$

El estudio histopatológico de las lesiones confirma el diagnóstico. En éste se observan glándulas y estroma endometrial entre los haces de tejido conectivo. Las glándulas tubulares se aprecian parcialmente ensanchadas y recubiertas de epitelio columnar. El estroma celular muestra áreas de edema, con algunos linfocitos y hemosiderófagos. ${ }^{4,10}$

Para completar el protocolo de estudio en una paciente con estas características se debe solicitar también una biometría hemática para determinar la presencia de anemia o alguna causa infecciosa que estuviese generando alteración menstrual, así como el impacto de la hipermenorrea en la endometriosis.

El diagnóstico diferencial clínico se debe realizar con hernias, lipomas, tumor desmoide o malignidad primaria o secundaria. La sospecha clínica preoperatoria es baja, por lo que conocer esta entidad ayuda al dermatólogo a realizar un diagnóstico temprano y proponer un tratamiento oportuno. ${ }^{4,8}$

El tratamiento incluye varias opciones, que pueden ser desde de la simple observación, el tratamiento quirúrgico, el puramente médico y/o combinación de alguno de éstos. ${ }^{5,7}$ Éste se debe individualizar con base

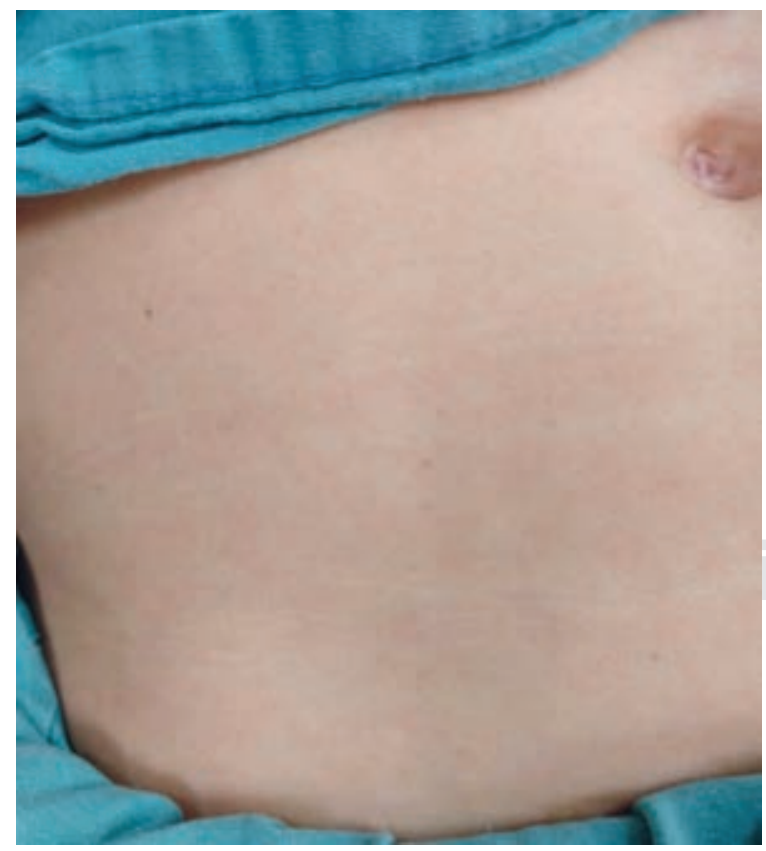

Figura 1: Imagen clínica de la superficie cutánea sin alteración aparente.

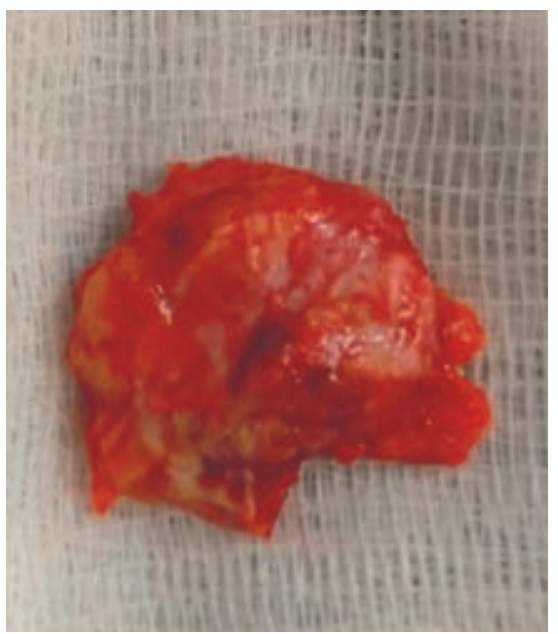

Figura 2:

En la pieza quirúrgica se observa tejido adiposo con grumos de material violáceo, de aspecto granuloso y friable.

en la gravedad de los síntomas y el deseo de fertilidad. En cuanto al tratamiento médico, se busca bloquear la función ovárica, creando un ambiente hipoestrogénico. Se utilizan anticonceptivos orales combinados (ACO) y progestágenos. Si fallan éstos, se inicia con el empleo de los agonistas $\mathrm{GnRH}$, o danazol, o los modificadores selectivos de los receptores estrogénicos (SERMs, por sus siglas en inglés), o los inhibidores de la aromatasa, entre otros. Como última alternativa se reserva el tratamiento quirúrgico. Con el fin de mejorar la calidad de vida de las pacientes, se indica la administración de todo lo anterior junto con analgésicos y algunos otros medicamentos para el control de los síntomas.

El pronóstico de la enfermedad depende de los sitios involucrados y la gravedad de los síntomas. La recurrencia de las lesiones después del tratamiento quirúrgico es de hasta $15 \% .{ }^{3,4}$ Los indicadores de una extirpación incompleta son la formación de seromas en el sitio del procedimiento quirúrgico y/o el inicio temprano del dolor referido previamente durante el postoperatorio inmediato. ${ }^{4,8}$

\section{CASO CLÍNICO}

Se trata de una mujer de 33 años de edad, quien acude a consulta general de dermatología refiriendo la presencia de una masa en abdomen desde hace siete años, dolorosa desde hace un año. Además, señala que en ocasiones ese dolor coincide con la presentación de su periodo menstrual. Entre sus antecedentes personales patológicos refiere haber cursado con un embarazo y una operación cesárea electiva ocho años y medio atrás. El resto de los antecedentes sin importancia para el padecimiento actual. 


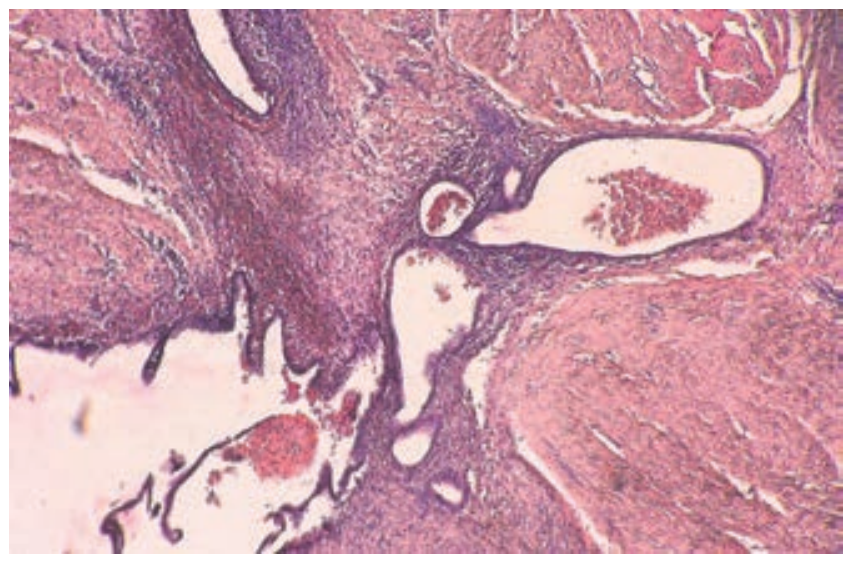

Figura 3: Se observan varias luces vasculares y glandulares inmersas en un estroma fibroso, (H\&E 4X).

A la exploración física se observa una dermatosis localizada en tronco, del cual afecta el abdomen en región periumbilical derecha que se extiende hasta hipocondrio derecho, unilateral. Constituida por una neoformación subcutánea, más palpable que visible, de bordes mal definidos, superficie lisa, móvil, no adherida a planos profundos, de consistencia blanda. De evolución crónica, dolorosa (Figura 1).

Con la impresión diagnóstica de un probable lipoma versus endometrioma se envía al servicio de cirugía dermatológica de la unidad para valoración, en donde se le programa para extirpación de la lesión. Durante el procedimiento se realiza disección hasta llegar a tejido celular subcutáneo profundo y fascia abdominal. Al llegar a este plano anatómico, se observa una masa amorfa fibrosa en la cual no se distinguen bordes (Figura 2). Se envía la pieza extraída al servicio de dermatopatología. El resultado del estudio histopatológico mostró tejido conjuntivo con amplias zonas de fibrosis e incremento en el número de fibroblastos. Entre las fibras de colágeno se aprecian numerosas cavidades de tipo glandular, todas ellas revestidas por una delgada pared de células secretoras de tipo apócrino. Se observan restos celulares, eritrocitos y fibrina en el interior de las cavidades, así como moderada reacción inflamatoria en el estroma circundante (Figuras 3 y 4). El diagnóstico histopatológico fue sugestivo de endometriosis.

La paciente fue valorada al día siguiente del procedimiento. Refería sólo dolor moderado en el sitio de la herida, el cual desapareció a los siete días, herida sin complicaciones, en vías de cicatrización. Se le refiere al servicio de ginecología en donde se le realiza un ultrasonido endovaginal, cuyos hallazgos reportan que la paciente cursa con endometriosis pélvica, al momento actual en manejo conservador, sólo con vigilancia, sin medicamentos, actualmente en seguimiento por cirugía dermatológica y ginecología.
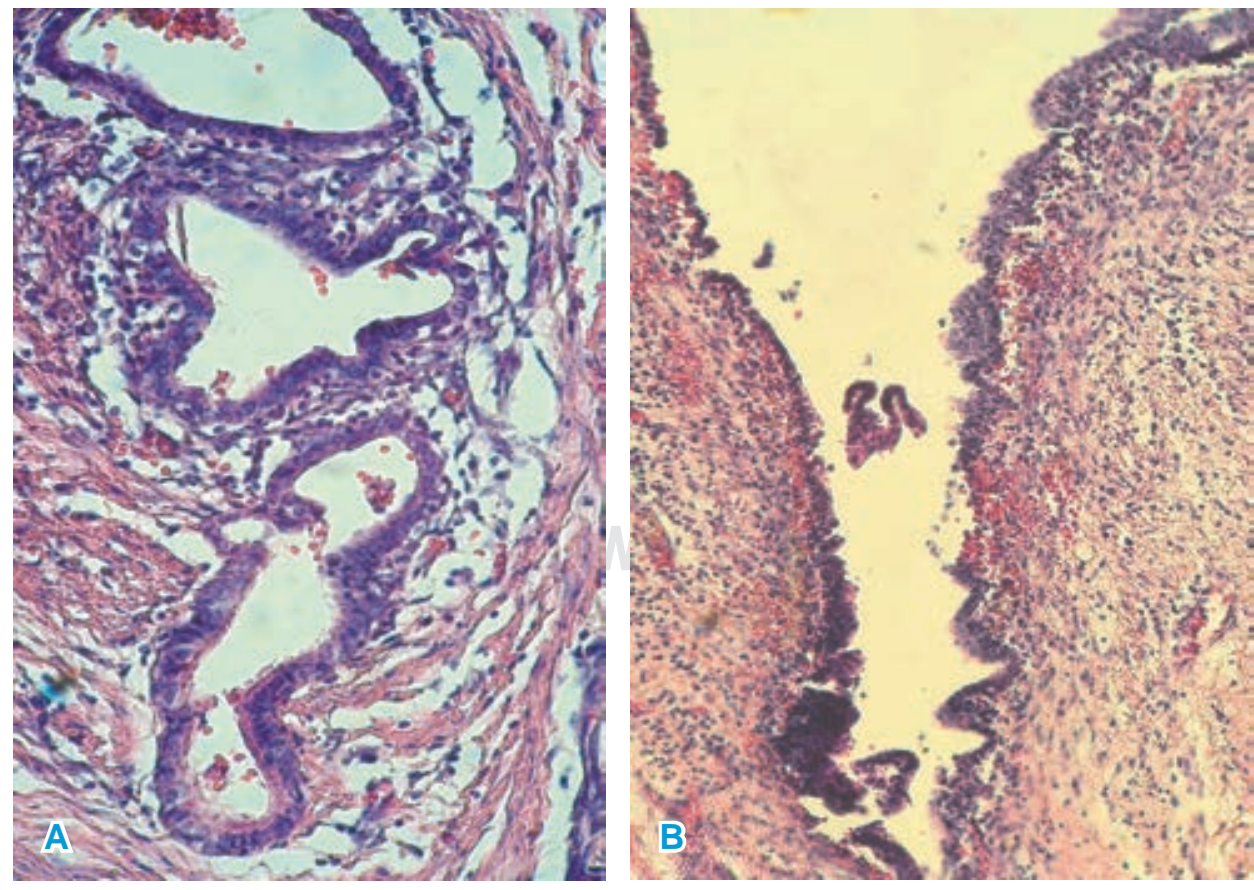

Figura 4:

A y B: Las luces ductales están revestidas por células que exhiben secreción por decapitación. Pequeños focos de hemorragia (H\&E 10X). 


\section{CONCLUSIÓN}

La endometriosis extrapélvica es una enfermedad rara que puede manifestarse como una masa subcutánea palpable en la pared abdominal. Puede o no cursar con síntomas típicos, por lo cual el caso que se comunica en este artículo es relevante en la práctica dermatológica, ya que su manifestación debe tenerse en cuenta para realizar diagnósticos diferenciales con otras entidades.

Por lo anterior, si una mujer en edad reproductiva acude con una masa abdominal subcutánea, dolor pélvico y con el antecedente de haber sido sometida a un procedimiento quirúrgico, debemos sospechar que se trata de un endometrioma cutáneo para así proporcionar un tratamiento oportuno, disminuyendo la morbilidad que causa esta enfermedad en mujeres en este grupo etario, ya que la endometriosis puede ocasionar dolor pélvico crónico e infertilidad.

\section{REFERENCIAS}

1. Montalto M, Santoro L, D'Onofrio F, Gallo A, Campo S, Campo V et al. Endometriosis, need for a multidisciplinary clinical setting: the internist's point of view. Intern Emerg Med. 2010; 5: 463-467.

2. Bauta-Desdin JL, Pupo-Zuñigas AM. Presentación de un paciente con endometriosis del canal anal. CCM [En línea]. 2016;20:826-833. Disponible en: http://scielo.sld.cu/scielo.php?script=sci_arttext\&pid $=$ S1560-43812016000400021.
3. Ulett-Araya NM. Actualización en los puntos clave de la endometriosis. Rev Méd Sinerg. 2019; 4: 35-43.

4. Dwivedi AJ, Agrawal SN, Silva YJ. Abdominal wall endometriomas. Dig Dis Sci. 2002; 47: 456-461.

5. Davis AC, Goldberg JM. Extrapelvic endometriosis. Semin Reprod Med. 2017; 35: 98-101.

6. Andres MP, Arcoverde FVL, Souza CCC, Fernandes LFC, Abrão MS, Kho RM. Extrapelvic endometriosis: a systematic review. J Minim Invasive Gynecol. 2020; 27: 373-389.

7. Machairiotis N, Stylianaki A, Dryllis G, Zarogoudilis P, Kouroutou P, Tsiamis $\mathrm{N}$ et al. Extrapelvic endometriosis: a rare entity or an under diagnosed condition? Diagn Pathol. 2013; 8: 194. Available in: https:// www.ncbi.nlm.nih.gov/pmc/articles/PMC3942279/.

8. Vagholkar K, Vagholkar S. Abdominal wall endometrioma: a diagnostic enigma-a case report and review of the literature. Case Rep Obstet Gynecol. 2019; 2019: 6831545. Available in: https://www.ncbi.nlm. nih.gov/pmc/articles/PMC6457300/.

9. Foti PV, Farina R, Palmucci S, Vizzini IAA, Libertini N, Coronella $\mathrm{M}$ et al. Endometriosis: clinical features, MR imaging findings and pathologic correlation. Insights Imaging. 2018; 9: 149-172.

10. Harzif AK, Silvia M, Mariana A, Olivia L, Lovita BT, Wiweko B. Extrapelvic endometriosis in abdominal wall scar and PPAR gamma expression: a case report. Int J Surg Case Rep. 2018; 53: 66-69.

\section{Correspondencia:}

Dr. Julio Enríquez Merino,

Dr. Vértiz Núm. 464, Esq. Eje 3 Sur,

Col. Buenos Aires, 06780,

Alcaldía Cuauhtémoc, CDMX.

Tel. 55 5519-6351.

E-mail: enriquez_drqx@yahoo.com.mx 